\title{
THE UNIT ROOT PROPERTY AND OPTIMALITY: \\ A SIMPLE PROOF*
}

\author{
Subir Chattopadhyay **
}

* I thank P. Gottardi, A. Jiménez and I. Zilcha for helpful comments. I gratefully
acknowledge financial support form CONACYT via a Cátedra de Excelencia at El
Colegio de México, additional support from the Ivie and DGICyT project PB97-0131
and the hospitality of ColMex.

${ }^{* *}$ S. Chattopadhyay: University of Alicante. 


\title{
THE UNIT ROOT PROPERTY AND OPTIMALITY: A SIMPLE PROOF
}

\author{
Subir Chattopadhyay
}

\begin{abstract}
A B S T R A C T
Consider a pure exchange OLG economy under stationay Markov uncertainty with one good and with sequentially complete markets. It is known that an interior stationary equilibrium allocation at which the agents common matrix of intertemporal rates of substitution has a Perron root which is less than or equal to one is conditionally Pareto optimal. We provide a simple and direct proof of this fact.
\end{abstract}

KEYWORDS: Stochastic Overlapping Generations Models; Pareto Optimality. 


\section{INTRODUCTION}

Consider a pure exchange, two-period lifetime overlapping generations (OLG) economy under stationary Markov uncertainty in which one good is available for consumption in each period. The stationary equilibria of such models when markets are assumed to be sequentially complete (so that an agent can trade against all risks faced in the second period of her life but not against the risk of being born in a bad state) are of interest since they provide a vehicle for the analysis of a variety of macroeconomic phenomenon, social security systems being the most important example. ${ }^{1}$ Because of their importance as good models, the optimality properties of such equilibria have become the focus of recent research. The following result (with minor variations) has been obtained with a finite number of Markov states: an interior stationary equilibrium allocation is Conditionally Pareto Optimal (CPO) if and only if it satisfies the unit root property which requires that the Perron root of the agents' common matrix of marginal rates of substitution, where each row of the matrix corresponds to an agent born in a different Markov state, is less than or equal to one. ${ }^{2}$

Aiyagari and Peled (1991) restricted attention to stationary reallocations only and obtained the result in a model with a stochastic linear technology. Chattopadhyay and Gottardi (1999) considered a general multigood pure exchange economy and allowed for all possible reallocations and obtained the result by using a general sufficient condition for equilibrium allocations to be CPO which they develop (their Theorem 3 ). ${ }^{3}$ These two papers have in common the feature that the analysis of optimality starts at a certain finite date and not at some ethereal "minus infinity". The implication is twofold; on the one hand they need to deal with a special agent(s), the "initial old", and on the other hand the set of agents in their economy is countable since at each date there are $S^{t}$ agents, where $S$ is the (finite) number of Markov states. An alternative specification, used by Zilcha (1991) and Demange and Laroque (1999), invokes a notion of stationarity which requires time to go to minus infinity; as a result their are no "initial old" and the set of agents can be identified with a continuum. The necessary and sufficient condition for CPO now requires that the Perron root be exactly equal to one; it is easy to see that this happens precisely because there are no initial old agent(s). The method of proof used by Demange and Laroque (1999) is based on considering the set of agents to be a nonatomic measure space so that in order to have a Pareto improvement it suffices to improve a set of agents of full measure; in fact, the proof relies heavily on probabilistic methods.

\footnotetext{
${ }^{1}$ The market can be sequentially complete because there are enough assets; also, if there is only one agent in each generation, as is often assumed, then the market is effectively sequentially complete.

${ }^{2}$ CPO, introduced by Muench (1977), is an optimality criterion which treats agents born in different events as distinct individuals.

${ }^{3}$ It is not difficult to see that the consideration of stochastic production does not change the basic result since the important issue is that of intertemporal optimality. As long as production decisions satisfy the conditions for optimal intratemporal resource allocation, which they will if spot markets are complete, they do not add anything of interest to the issue of intertemporal optimality even though a model with production appears to be more realistic.
} 
It is reasonable to ask whether a notion of stationarity with time going to minus infinity is appealing when considering welfare comparisons. It is well known that in the case of a deterministic economy such a notion of stationarity picks out the Golden Rule as the unique stationary optimal allocation while a continuum of stationary allocations would be considered optimal when the welfare of the initial old is taken into account. We believe that even though economic equilibrium can be considered to have been established at an ethereal minus infinity, any analysis of optimality must start at a finite date with a given history. Doing so leaves us with a countable set of agents to which we wish to apply the Pareto criterion; now it is no longer obvious that improving every agent in a set of full measure is useful as a welfare criterion.

By considering a second order approximation to an agent's utility at the competitive allocation, we provide a direct and simple proof of the unit root property which applies to a countable set of agents. Our proof is nonprobabilistic and is a direct generalization of the classroom proof of the optimality of the Golden Rule in deterministic economies; as a consequence it is much simpler than the proof in Chattopadhyay and Gottardi (1999) as it directly exploits the stationary structure of the equilibrium. ${ }^{4}$

\section{THE MODEL}

We consider a one good, two period lifetime, pure exchange overlapping generations (OLG) economy under stationary Markov uncertainty. We turn to a formal description of the model and the notation used. ${ }^{5}$

Time is discrete and dates are denoted by $t=1,2,3, \cdots$.

Let $\mathcal{S}$ be the state space of the Markov process with $S:=\# \mathcal{S}<\infty$. The structure of the date-event tree induced by all possible realizations of states from an initial date $t=0$ is as follows. The root of the tree is $\sigma_{0} \in \mathcal{S}$; the set of nodes at date $t$ is denoted $\Sigma_{t}$ where we set $\Sigma_{1}:=\left\{\sigma_{0}\right\} \times \mathcal{S}$, and, iteratively, set $\Sigma_{t}:=\Sigma_{t-1} \times \mathcal{S}$ for $t=2,3, \cdots$. Define $\Sigma:=\cup_{t \geq 1} \Sigma_{t}$ and $\Gamma:=\left\{\sigma_{0}\right\} \cup \Sigma$. Elements of $\Gamma$ are called nodes (to be thought of as the "date-events" or simply "events"), and a generic node is denoted by $\sigma$. Given a node $\sigma \in \Sigma, t(\sigma)$ denotes the value of $t$ at which $\sigma \in \Sigma_{t}$, and $s(\sigma)$ identifies the Markov state. Clearly, a node $\sigma \in \Sigma_{t}$ is nothing but a string $\left(\sigma_{0}, s_{1}, s_{2}, \cdots, s_{t}\right)$, where $s_{\tau} \in \mathcal{S}$ denotes the realization of the process at date $\tau, \tau=1, \cdots, t\left(\sigma_{0}\right.$ is the realization at the initial date). It follows that the predecessor of a node $\sigma \in \Sigma_{t}$ is uniquely defined and it will be denoted by $\sigma_{-1}$, an element of $\Sigma_{t-1}$; the set of immediate successor nodes of a node $\sigma$ is denoted $\sigma^{+}$.

One commodity is available for consumption at each node $\sigma \in \Sigma$.

At each node $\sigma \in \Sigma, \mathcal{H}$, a generation of agents, is born, where $H:=\# \mathcal{H}$. Each agent

\footnotetext{
${ }^{4}$ Theorem 3 in Chattopadhyay and Gottardi (1999) follows the method pioneered by Cass (1972) for the analysis of efficient production paths under certainty, and provides a general sufficient condition, in the form of a Cass criterion, for equilibria to be CPO. As they show, the criterion in their Theorem 3 is not a necessary condition in general but for stationary equilibria the condition turns out to be necessary and sufficient.

${ }^{5}$ We use the notation developed in Chattopadhyay and Gottardi (1999).
} 
lives at two dates. The consumption plan of an agent specifies the level of consumption in the event at birth and in its immediate successor nodes. A member of generation $\sigma$ of type $h \in \mathcal{H}$ is denoted by $(\sigma, h)$.

In addition, there is a set, $\mathcal{H}$, of $H$ one-period lived agents who enter the economy at each node $\sigma \in \Sigma_{1}$ at date 1; they constitute the generation of the "initial old", and are indexed by $(\sigma, h, o)$, where $\sigma \in \Sigma_{1}$.

We will assume that the economy is stationary, i.e., that the characteristics (consumption sets, endowments, and utility functions) of each agent depend only on the realizations of the Markov state during her lifetime, not on time nor on past realizations. So, for any $(\sigma, \widehat{\sigma}) \in \Sigma \times \Sigma, s(\sigma)=s(\widehat{\sigma})$ implies that (i) for consumption sets $X_{\sigma, h}=X_{\hat{\sigma}, h}:=X_{s(\sigma), h}$, (ii) for endowments $\omega(\sigma, h)=\omega(\widehat{\sigma}, h):=\omega(s(\sigma), h)$, where $\omega(s, h)=\left(\omega(s ; s, h),\left(\omega\left(s, s^{\prime} ; s, h\right)\right)_{s^{\prime} \in \mathcal{S}}\right)$ describes the endowment at birth and in all successor nodes, and (iii) for utility functions $u_{\sigma, h}=u_{\hat{\sigma}, h}:=u_{s(\sigma), h}$ (for the initial old we use the notation $X_{s(\sigma), h, o}, \omega(s(\sigma) ; h, o)$, and $\left.u_{s(\sigma), h, o}\right)$. Let $\omega(s ; h, o)=\omega(\tilde{s}, s ; \tilde{s}, h)$ for all $s \in \mathcal{S}$ and for all $h \in \mathcal{H}$ for some $\tilde{s} \in \mathcal{S}$; this lets us introduce the initial old in a manner which is compatible with the stationary structure of the rest of the economy.

A consumption plan for agent $(\sigma, h)$ will be denoted by $x(\sigma, h)=\left(x(\sigma ; \sigma, h),\left(x\left(\sigma^{\prime} ; \sigma, h\right)\right)_{\sigma^{\prime} \in \sigma^{+}}\right)$ $(x(\sigma ; h, o)$ for the initial old $)$; this notation allows us to consider nonstationary consumption plans even though the environment is stationary.

Denoting by $\omega(\sigma)$ the total endowment at node $\sigma$, we have:

$\omega(\sigma):=\sum_{h \in \mathcal{H}} \omega(s(\sigma) ; s(\sigma), h)+\sum_{h \in \mathcal{H}} \omega(s(\sigma) ; h, o) \quad$ for $\sigma \in \Sigma_{1}$,

$\omega(\sigma):=\sum_{h \in \mathcal{H}} \omega(s(\sigma) ; s(\sigma), h)+\sum_{h \in \mathcal{H}} \omega\left(s\left(\sigma_{-1}\right), s(\sigma) ; s\left(\sigma_{-1}\right), h\right) \quad$ for $\quad \sigma \in \cup_{t \geq 2} \Sigma_{t}$.

We impose the following standard conditions:

\section{ASSUMPTION 1:}

(i) $1 \leq H<\infty$ and $1 \leq \# \mathcal{S}:=S<\infty$.

(iia) For all $(s, h, o) \in \mathcal{S} \times \mathcal{H}, X_{s, h, o}=R_{+}, \quad u_{s, h, o}: X_{s, h, o} \rightarrow R$ is strictly monotone.

(iib) For all $(s, h) \in \mathcal{S} \times \mathcal{H}, X_{s, h}=R_{+}^{1+S}, \omega(s ; s, h) \in R_{++}$and $\left(\left(\omega\left(s, s^{\prime} ; s, h\right)\right)_{s^{\prime} \in \mathcal{S}}\right) \in$ $R_{+}^{S} /\{\overline{0}\}, \quad u_{s, h}: X_{s, h} \rightarrow R$ is $C^{2}$, strictly monotone, and differentiably strictly quasiconcave.

(iii) $\omega(s ; h, o)=\omega(\tilde{s}, s ; \tilde{s}, h)$ for all $s \in \mathcal{S}$ and for all $h \in \mathcal{H}$ for some $\tilde{s} \in \mathcal{S}$.

(iv) For all $\sigma \in \Sigma, \omega(\sigma) \in R_{++}$.

DEFINITION 1: A feasible allocation $x$ is given by an array

$\left((x(\sigma ; h, o))_{(\sigma, h) \in \Sigma_{1} \times \mathcal{H}},(x(\sigma, h))_{(\sigma, h) \in \Sigma \times \mathcal{H}}\right)$ such that $x(\sigma ; h, o) \in X_{s(\sigma), h, o}$ for all $(\sigma, h) \in$ $\Sigma_{1} \times \mathcal{H}, x(\sigma, h) \in X_{s(\sigma), h}$ for all $(\sigma, h) \in \Sigma \times \mathcal{H}$, and

$\sum_{h \in \mathcal{H}} x(\sigma ; \sigma, h)+\sum_{h \in \mathcal{H}} x(\sigma ; h, o) \leq \omega(\sigma)$ for all $\sigma \in \Sigma_{1}$,

$\sum_{h \in \mathcal{H}} x(\sigma ; \sigma, h)+\sum_{h \in \mathcal{H}} x\left(\sigma ; \sigma_{-1}, h\right) \leq \omega(\sigma)$ for all $\sigma \in \cup_{t \geq 2} \Sigma_{t}$.

We use a notion of optimality proposed by Muench (1977) wherein the Pareto criterion 
is applied to an economy in which agents are distinguished by the event at their birth:

DEFINITION 2 (CPO): Let $x$ be a feasible allocation. $x$ is conditionally Pareto optimal $(C P O)$ if there does not exist another feasible allocation $\widehat{x}$ such that

(i) for all $(\sigma, h) \in \Sigma_{1} \times \mathcal{H}, u_{s(\sigma), h, o}(\widehat{x}(\sigma ; h, o)) \geq u_{s(\sigma), h, o}(x(\sigma ; h, o))$, for all $(\sigma, h) \in \Sigma \times \mathcal{H}, u_{s(\sigma), h}(\widehat{x}(\sigma, h)) \geq u_{s(\sigma), h}(x(\sigma, h))$,

(ii) either for some $\left(\sigma^{\prime}, h^{\prime}\right) \in \Sigma_{1} \times \mathcal{H}, u_{s\left(\sigma^{\prime}\right), h^{\prime}, o}\left(\widehat{x}\left(\sigma^{\prime} ; h^{\prime}, o\right)\right)>u_{s\left(\sigma^{\prime}\right), h^{\prime}, o}\left(x\left(\sigma^{\prime} ; h^{\prime}, o\right)\right)$, or for some $\left(\sigma^{\prime}, h^{\prime}\right) \in \Sigma \times \mathcal{H}, u_{s\left(\sigma^{\prime}\right), h^{\prime}}\left(\widehat{x}\left(\sigma^{\prime}, h^{\prime}\right)\right)>u_{s\left(\sigma^{\prime}\right), h^{\prime}}\left(x\left(\sigma^{\prime}, h^{\prime}\right)\right)$.

We now introduce the notion of stationary equilibrium when markets are sequentially complete. Let $q_{s}=\left(\left(q_{s, s^{\prime}}\right)_{s^{\prime} \in \mathcal{S}}\right) \in R_{+}^{S}$ denote the stationary price of a one period ahead contingent claim on the consumption good bought in the state $s \in \mathcal{S}$. Let $q:=\left(q_{1}, \cdots, q_{S}\right)$ be the vector of stationary prices.

Stationarity of the equilibrium requires that $x(\sigma, h)=x(s(\sigma), h)$ for all $(\sigma, h) \in \Sigma \times \mathcal{H}$ (i.e., the consumption allocation of each agent depends on the state at the date of his birth and the states at the next date only); a stationary consumption plan for agent $(\sigma, h)$, with $s=s(\sigma)$, will be denoted by $x(s, h)=\left(x(s ; s, h),\left(x\left(s, s^{\prime} ; s, h\right)\right)_{s^{\prime} \in \mathcal{S}}\right)$. We also need to assign consumption to the initial old; for our purposes it suffices that the allocation be feasible in the aggregate since Assumption 1 (iii) guarantees that the aggregate endowment is stationary. ${ }^{6}$

DEFINITION 3: $\left(x^{*}, q^{*}\right)$ is a stationary competitive equilibrium with sequentially complete markets if $x^{*}$ is a feasible allocation and, for all $(s, h) \in \mathcal{S} \times \mathcal{H}$,

(a) $\left[x^{*}(s ; s, h)-\omega(s ; s, h)\right]+\sum_{s^{\prime} \in \mathcal{S}} q_{s, s^{\prime}}^{*} \cdot\left[x^{*}\left(s, s^{\prime} ; s, h\right)-\omega\left(s, s^{\prime} ; s, h\right)\right] \leq 0$,

(b) if $u_{s, h}(x)>u_{s, h}\left(x^{*}(s, h)\right)$ then

$[x(s ; s, h)-\omega(s ; s, h)]+\sum_{s^{\prime} \in \mathcal{S}} q_{s, s^{\prime}}^{*} \cdot\left[x\left(s, s^{\prime} ; s, h\right)-\omega\left(s, s^{\prime} ; s, h\right)\right]>0$.

\section{THE RESULT}

At an interior equilibrium allocaton, let $m_{s, s^{\prime}, h}\left(x^{*}\right):=\frac{d u_{s, h}\left(x^{*}(s, h)\right)}{d x_{1+s^{\prime}}} / \frac{d u_{s, h}\left(x^{*}(s, h)\right)}{d x_{1}} ;^{7}$ let $M_{h}\left(x^{*}\right)$ be the strictly positive square matrix with elements $m_{s, s^{\prime}, h}\left(x^{*}\right)$. Since markets are sequentially complete, the matrices $M_{h}\left(x^{*}\right)$ are identical for all the agents; let $M\left(x^{*}\right)$, a strictly positive matrix, be the common value of the matrices. By Perron's Theorem (see, e.g., Theorem 8.2.8 in Horn and Johnson (1985)), there exists a unique vector (up to normalization) with all components positive, $y \in R_{++}^{S}$, such that $M\left(x^{*}\right) \cdot y=\lambda \cdot y$ for some number $\lambda \in R_{++}$. The number $\lambda$ is the Perron root of the matrix $M\left(x^{*}\right)$ and is the largest eigenvalue, in absolute value, of the matrix. The equilibrium is said to satisfy the unit root property if the Perron root of the matrix $M\left(x^{*}\right)$ is less than or equal to one.

\footnotetext{
${ }^{6}$ A complete specification of equilibrium requires the introduction of (i) commodity transfers via taxes, if we think in terms of trade taking place in contingent commodities, or (ii) assets, if we think of trade taking place in a sequence of markets, which support the net trade between generations required by the prices $q$. Either of these specifies completely the consumption of the initial old.

${ }^{7}$ For $f: R_{++}^{N} \rightarrow R, \frac{d f(\bar{x})}{d x_{i}}$ denotes the partial derivative of the function $f$ with respect to its $i$-th coordinate evaluated at the point $\bar{x}$.
} 
THEOREM: Let $\left(x^{*}, q^{*}\right)$ be a stationary competitive equilibrium with sequentially complete markets in which the equilibrium allocation is interior. Under Assumption 1, the equilibrium allocation is CPO if and only if it satisfies the unit root property.

PROOF: That there exists a stationary improvement when the Perron root exceeds one can be seen as follows. ${ }^{8}$ By the defining property of the Perron root of the Matrix $M\left(x^{*}\right)$ there exists a vector $y \in R_{++}^{S}$ such that

$$
\begin{gathered}
\lambda y=M\left(x^{*}\right) \cdot y \quad \Leftrightarrow \quad \lambda y_{s}=\sum_{s^{\prime} \in \mathcal{S}} m_{s, s^{\prime}}\left(x^{*}\right) y_{s^{\prime}} \quad \text { for all } s \in \mathcal{S} \\
\Leftrightarrow \quad \sum_{s^{\prime} \in \mathcal{S}} \frac{d u_{s, h}\left(x^{*}(s, h)\right)}{d x_{1+s^{\prime}}} y_{s^{\prime}}+\frac{d u_{s, h}\left(x^{*}(s, h)\right)}{d x_{1}}\left(-y_{s}\right) \lambda=0 \quad \text { for all } s \in \mathcal{S} \\
\Leftrightarrow \quad \sum_{s^{\prime} \in \mathcal{S}} \frac{d u_{s, h}\left(x^{*}(s, h)\right)}{d x_{1+s^{\prime}}} y_{s^{\prime}}+\frac{d u_{s, h}\left(x^{*}(s, h)\right)}{d x_{1}}\left(-y_{s}\right)>0 \quad \text { for all } s \in \mathcal{S},
\end{gathered}
$$

since $\lambda>1$, where $h$ is any agent type. So by reducing the consumption of an agent born in state $s$ by $y_{s}$ and increasing it by $y_{s^{\prime}}$ in each state when old, one generates an improvement. Feasibility is obviously maintained. Since the transfers proposed are from the young to the old, the initial old are also improved in every state.

The argument above makes clear that if the unit root property holds there can be no stationary improvement; we argue by contradiction to show that there are no nonstationary improvements either.

Suppose there exists an allocation $\hat{x}$ that CPO dominates $x^{*}$. Define

$$
\begin{gathered}
\Delta(\sigma ; \sigma, h):=\hat{x}(\sigma ; \sigma, h)-x^{*}(s(\sigma) ; s(\sigma), h), \quad \text { for } \sigma \in \Sigma, \\
\Delta\left(\sigma^{\prime} ; \sigma, h\right):=\hat{x}\left(\sigma^{\prime} ; \sigma, h\right)-x^{*}\left(s(\sigma), s\left(\sigma^{\prime}\right) ; s(\sigma), h\right), \quad \text { for } \sigma^{\prime} \in \sigma^{+},
\end{gathered}
$$

where we use notation that allows us to treat the case in which the allocation $\hat{x}$ is not stationary. Also define $\bar{\Delta}(\sigma):=-\frac{1}{H} \sum_{h \in \mathcal{H}} \Delta(\sigma ; \sigma, h)$, the change in the average consumption by the young born at the node $\sigma$. Feasibility of the alternative allocation implies that

$$
\frac{1}{H} \sum_{h \in \mathcal{H}} \Delta\left(\sigma^{\prime} ; \sigma, h\right)-\bar{\Delta}\left(\sigma^{\prime}\right) \leq 0, \quad \text { for } \sigma^{\prime} \in \sigma^{+} .
$$

It follows that if the change in average consumption by the old at a node $\sigma$ is positive then $\bar{\Delta}(\sigma)$ is also positive.

Since we have an improving allocation, and preferences are strictly convex, the following inequality, which gives a quadratic approximation to an agent's utility function around the equilibrium allocation, must be satisfied for all agents $(\sigma, h), \sigma \in \Sigma,{ }^{9}$

$$
\sum_{s^{\prime} \in \mathcal{S}} m_{s(\sigma), s^{\prime}}\left(x^{*}\right) \Delta\left(\sigma, s^{\prime} ; \sigma, h\right) \geq-\Delta(\sigma ; \sigma, h)+\underline{\rho} \cdot[\Delta(\sigma ; \sigma, h)]^{2},
$$

${ }^{8}$ This argument is due to Aiyagari and Peled (1991).

${ }^{9}$ See, e.g., Lemma 1 in Chattopadhyay and Gottardi (1999). 
where we identify the node $\sigma^{\prime} \in \sigma^{+}$with $\left(\sigma, s\left(\sigma^{\prime}\right)\right)$ which we denote $\left(\sigma, s^{\prime}\right)$. $\underline{\rho}$ is a positive number which gives a uniform lower bound on the curvature of the agents' indifference sets and is well defined under Assumption 1.

By averaging the inequality in (1) across the set of agents born at the same node, using Jensen's inequality applied to a quadratic function, and using the feasibility condition, we obtain:

$$
\sum_{s^{\prime} \in \mathcal{S}} m_{s(\sigma), s^{\prime}}\left(x^{*}\right) \bar{\Delta}\left(\sigma, s^{\prime}\right) \geq \bar{\Delta}(\sigma)+\underline{\rho} \cdot[\bar{\Delta}(\sigma)]^{2} .
$$

In addition, the fact that the initial old must also be improved implies that $\bar{\Delta}(\sigma) \geq 0$ for all $\sigma \in \Sigma_{1}$. This fact together with an application of (2) shows that there must be some node $\bar{\sigma}$ such that the change in average consumption by the young is zero for all agents born at nodes with dates $t(\sigma)<t(\bar{\sigma})$ while $\bar{\Delta}(\bar{\sigma})>0$.

Let $\lambda$ denote the Perron root of $M\left(x^{*}\right)$ and let $y \in R_{++}^{S}$ be a vector such that $M\left(x^{*}\right) \cdot y=\lambda \cdot y$, so that for all $s \in \mathcal{S}$ we have $\sum_{s^{\prime} \in \mathcal{S}} m_{s, s^{\prime}}\left(x^{*}\right) y_{s^{\prime}}=\lambda y_{s}>0$; it follows that by setting $w\left(s, s^{\prime}\right):=\frac{m_{s, s^{\prime}}\left(x^{*}\right) y_{s^{\prime}}}{\lambda y_{s}}$ we have $w\left(s, s^{\prime}\right)>0$, for all $\left(s, s^{\prime}\right) \in \mathcal{S} \times \mathcal{S}$, and $\sum_{s^{\prime} \in \mathcal{S}} w\left(s, s^{\prime}\right)=1$ for all $s \in \mathcal{S}$. (2) can now be rewritten as

$$
\lambda \sum_{s^{\prime} \in \mathcal{S}} w\left(s(\sigma), s^{\prime}\right) \frac{\bar{\Delta}\left(\sigma, s^{\prime}\right)}{y_{s^{\prime}}} \geq \frac{\bar{\Delta}(\sigma)}{y_{s(\sigma)}}+\underline{\rho} \cdot \frac{[\bar{\Delta}(\sigma)]^{2}}{y_{s(\sigma)}} .
$$

The existence of an improving allocation implies that (3) must hold.

To reduce the notational burden, we will assume that $\bar{\sigma} \in \Sigma_{1}$, that is, the initial old are the first to receive a transfer; this is without loss of generality. ${ }^{10}$

Define $W\left(\sigma_{0}\right):=1, W(\bar{\sigma}):=1, W(\sigma)=0$ for $\sigma \in \Sigma_{1} /\{\bar{\sigma}\}$, and inductively define $W(\sigma):=W\left(\sigma_{-1}\right) \cdot w\left(s\left(\sigma_{-1}\right), s(\sigma)\right)$ for $\sigma \in \cup_{t \geq 2} \Sigma_{t}$. It is easy to see that $\sum_{\sigma \in \Sigma_{t}} W(\sigma)=1$ for all $t \geq 1$ and that $W(\sigma)>0$ if and only if $\sigma$ is a node that succeeds $\bar{\sigma}$ which was defined as the first node at which the average intertemporal transfer is nonzero.

Evidently, invoking (3) twice yields

$$
\begin{gathered}
\lambda^{2} \sum_{s^{\prime} \in \mathcal{S}} \sum_{s^{\prime \prime} \in \mathcal{S}} w\left(s(\bar{\sigma}), s^{\prime}\right) w\left(s^{\prime}, s^{\prime \prime}\right) \frac{\bar{\Delta}\left(\left(\bar{\sigma}, s^{\prime}\right), s^{\prime \prime}\right)}{y_{s^{\prime \prime}}}=\lambda \sum_{s^{\prime} \in \mathcal{S}} w\left(s(\bar{\sigma}), s^{\prime}\right)\left\{\lambda \sum_{s^{\prime \prime} \in \mathcal{S}} w\left(s^{\prime}, s^{\prime \prime}\right) \frac{\bar{\Delta}\left(\left(\bar{\sigma}, s^{\prime}\right), s^{\prime \prime}\right)}{y_{s^{\prime \prime}}}\right\} \\
\geq \lambda \sum_{s^{\prime} \in \mathcal{S}} w\left(s(\bar{\sigma}), s^{\prime}\right)\left\{\frac{\bar{\Delta}\left(\bar{\sigma}, s^{\prime}\right)}{y_{s^{\prime}}}+\underline{\rho} \cdot \frac{\left[\bar{\Delta}\left(\bar{\sigma}, s^{\prime}\right)\right]^{2}}{y_{s^{\prime}}}\right\} \\
\geq \frac{\bar{\Delta}(\bar{\sigma})}{y_{s(\sigma)}}+\underline{\rho} \cdot\left\{\frac{[\bar{\Delta}(\bar{\sigma})]^{2}}{y_{s(\bar{\sigma})}}+\lambda \cdot \sum_{s^{\prime} \in \mathcal{S}} w\left(s(\sigma), s^{\prime}\right) \frac{\left[\bar{\Delta}\left(\bar{\sigma}, s^{\prime}\right)\right]^{2}}{y_{s^{\prime}}}\right\} .
\end{gathered}
$$

Equivalently, we have shown that

$$
\lambda^{2} \sum_{\sigma \in \Sigma_{3}} W(\sigma) \frac{\bar{\Delta}(\sigma)}{y_{s(\sigma)}} \geq \frac{\bar{\Delta}(\bar{\sigma})}{y_{s(\bar{\sigma})}}+\underline{\rho} \cdot\left\{\sum_{\tau=1}^{2} \lambda^{\tau-1} \sum_{\sigma \in \Sigma_{\tau}} W(\sigma) \cdot \frac{[\bar{\Delta}(\sigma)]^{2}}{y_{s(\sigma)}}\right\} .
$$

\footnotetext{
${ }^{10}$ We can always ignore the set of dates at which the net transfer is zero since once the transfer is positive at a node at some date it must be positive along some sequence of successor nodes.
} 
By repeating the argument we obtain a set of inequalities which must hold if an improvement exists:

$$
\lambda^{T} \sum_{\sigma \in \Sigma_{T+1}} W(\sigma) \cdot \frac{\bar{\Delta}(\sigma)}{y_{s(\sigma)}} \geq \frac{\bar{\Delta}(\bar{\sigma})}{y_{s(\bar{\sigma})}}+\underline{\rho} \cdot\left\{\sum_{\tau=1}^{T} \lambda^{\tau-1} \sum_{\sigma \in \Sigma_{\tau}} W(\sigma) \cdot \frac{[\bar{\Delta}(\sigma)]^{2}}{y_{s(\sigma)}}\right\} .
$$

Since the endowment is uniformly bounded across nodes and since $y \in R_{++}^{S}$, feasibility implies that the quantity $\frac{\bar{\Delta}(\sigma)}{y_{s(\sigma)}}$ is always bounded. Since $\sum_{\sigma \in \Sigma_{t}} W(\sigma)=1$ for all $t \geq 0$, the left hand side of the inequality is an expected value of a bounded variable multiplied by a growth factor. Clearly, if $\lambda<1$, the left hand side converges to zero while the right hand side is positive, as $\bar{\Delta}(\bar{\sigma})>0$, yielding a contradiction.

In order to proceed, note that for $w_{i}>0$ with $\sum_{i} w_{i}=1$, and $x_{i}$ arbitrary,

$$
\sum_{i} w_{i} x_{i} \geq a \geq 0 \quad \Rightarrow \quad \sum_{i} w_{i}\left(x_{i}\right)^{2} \geq a^{2}
$$

which follows from the fact that

$$
\sum_{i} w_{i}\left[x_{i}-\left(\sum_{i} w_{i} x_{i}\right)\right]^{2} \geq 0 \quad \Leftrightarrow \quad \sum_{i} w_{i}\left(x_{i}\right)^{2} \geq\left(\sum_{i} w_{i} x_{i}\right)^{2}
$$

Since (4), evaluated at $\lambda=1$, implies that

$$
\sum_{\sigma \in \Sigma_{t}} W(\sigma) \cdot \frac{\bar{\Delta}(\sigma)}{y_{s(\sigma)}} \geq \frac{\bar{\Delta}(\bar{\sigma})}{y_{s(\bar{\sigma})}}>0 \quad \text { for all } t=2,3, \cdots,
$$

an application of (5) shows that

$$
\sum_{\sigma \in \Sigma_{t}} W(\sigma) \cdot \frac{[\bar{\Delta}(\sigma)]^{2}}{\left[y_{s(\sigma)}\right]^{2}} \geq \frac{[\bar{\Delta}(\bar{\sigma})]^{2}}{\left[y_{s(\bar{\sigma})}\right]^{2}} \quad \Leftrightarrow \quad \sum_{\sigma \in \Sigma_{t}} W(\sigma) \cdot \frac{[\bar{\Delta}(\sigma)]^{2}}{y_{s(\sigma)}} \geq \frac{y}{\bar{y}} \cdot \frac{[\bar{\Delta}(\bar{\sigma})]^{2}}{y_{s(\bar{\sigma})}}
$$

where $y$ is the smallest element of the strictly positive vector $y$ and $\bar{y}$ is the largest element. Now it is obvious that in (4) with $\lambda=1$, the left hand side is bounded while the right hand side is not and this yields the desired contradiction. 


\section{REFERENCES}

AIYAGARI, S. R., AND D. PELED (1991): "Dominant Root Characterization of Pareto Optimality and the Existence of Optimal Monetary Equilibria in Stochastic OLG Models," Journal of Economic Theory, 54, 69-83.

CASS, D. (1972): "On Capital Overaccumulation in the Aggregative Neoclassical Model of Economic Growth: A Complete Characterization," Journal of Economic Theory, 4, 200-223.

CHATTOPADHYAY, S., AND P. GOTTARDI (1999): "Stochastic OLG Models, Market Structure, and Optimality," Journal of Economic Theory, 89, 21-67.

DEMANGE, G., AND G. LAROQUE (1999): "Social Security and Demographic Shocks," Econometrica, 67, 527-542.

HORN, R. A., AND C. R. JOHNSON (1985): "Matrix Analysis," Cambridge University Press, Cambridge.

MUENCH, T. J. (1977): "Optimality, the Interaction of Spot and Futures Markets, and the Non-neutrality of Money in the Lucas Model," Journal of Economic Theory, 15, 325344.

ZILCHA, I. (1991): "Characterizing Efficiency in Stochastic Overlapping Generations Models," Journal of Economic Theory, 55, 1-16. 